\title{
Principal Eigenvalues of a Second-Order Difference Operator with Sign-Changing Weight and Its Applications
}

\author{
Ruyun Ma (D), Man Xu, and Yan Long \\ Department of Mathematics, Northwest Normal University, Lanzhou 730070, China \\ Correspondence should be addressed to Ruyun Ma; mary@nwnu.edu.cn
}

Received 31 October 2017; Revised 14 December 2017; Accepted 24 December 2017; Published 18 January 2018

Academic Editor: Douglas R. Anderson

Copyright (C) 2018 Ruyun Ma et al. This is an open access article distributed under the Creative Commons Attribution License, which permits unrestricted use, distribution, and reproduction in any medium, provided the original work is properly cited.

Let $T>2$ be an integer and $\mathbb{T}=\{1,2, \ldots, T\}$. We show the existence of the principal eigenvalues of linear periodic eigenvalue problem $-\Delta^{2} u(j-1)+q(j) u(j)=\lambda g(j) u(j), j \in \mathbb{T}, u(0)=u(T), u(1)=u(T+1)$, and we determine the sign of the corresponding eigenfunctions, where $\lambda$ is a parameter, $q(j) \geq 0$ and $q(j) \neq \equiv$ in $\mathbb{T}$, and the weight function $g$ changes its sign in $\mathbb{T}$. As an application of our spectrum results, we use the global bifurcation theory to study the existence of positive solutions for the corresponding nonlinear problem.

\section{Introduction}

In 1997, Constantin [1] studied the following linear periodic eigenvalue problem:

$$
\begin{aligned}
u^{\prime \prime}(t)-q(t) u(t) & =\lambda m(t) u(t), \quad t \in(0, w), \\
u(0) & =u(w), \\
u^{\prime}(0) & =u^{\prime}(w),
\end{aligned}
$$

where $m, q \in C[0, w], q(t) \geq 0$, and $q(t) \not \equiv 0$. He obtained that if $m$ changes its sign, then (1) and (2) have infinite real eigenvalues, $\lambda_{k}^{ \pm}$, such that

$$
\cdots \leq \lambda_{3}^{-} \leq \lambda_{2}^{-}<\lambda_{1}^{-}<0<\lambda_{1}^{+}<\lambda_{2}^{+} \leq \lambda_{3}^{+} \leq \cdots .
$$

Equation (1) with $q \equiv 1 / 4$ plays a crucial role in the study of the water shallow equation; see [2-5].

Let $T>2$ be an integer and $\mathbb{T}=\{1,2, \ldots, T\}$. In 2005, Wang and Shi [6] discussed the eigenvalues of a discrete periodic boundary value problem

$$
\begin{aligned}
& \Delta[p(j-1) \Delta u(j-1)]+q(j) u(j)+\lambda m(j) u(j) \\
& \quad=0, \quad j \in \mathbb{T}, \\
& u(0)=u(T), \\
& u(1)=u(T+1),
\end{aligned}
$$

where $p(j), q(j)$, and $m(j)$ are real functions with $p(j)>0$ for $j \in\{0,1, \ldots, T\}, m(j)>0$ for $j \in \mathbb{T}$, and $p(0)=p(T)=1$, and $\lambda$ is the spectral parameter. They showed the existence of eigenvalues of (4) and (5) and calculated the numbers of eigenvalues.

Ji and Yang [7] considered a class of boundary value problems of the second-order difference equation (4) with the more general boundary conditions

$$
\begin{aligned}
u(0) & =\alpha u(T), \\
u(T+1) & =\alpha u(1) .
\end{aligned}
$$

The class of problems considered include those with antiperiodic, Dirichlet, and periodic boundary conditions. They focused on the structure of eigenvalues and comparisons of all eigenvalues of (4) and (6), as the coefficients $p(t), q(t)$, and $m(t)$ change their signs. They got a very interesting result: the numbers of positive eigenvalues are equal to the numbers of positive elements in the weight function, and the numbers of negative eigenvalues are equal to the numbers of negative elements in the weight function.

Gao and Ma [8] studied the eigenvalues of periodic and antiperiodic eigenvalue problems of discrete linear secondorder difference equation (4) with sign-changing weight. They find that these two problems have $T$ real eigenvalues (including the multiplicity), respectively. Furthermore, the 
numbers of positive eigenvalues are equal to the numbers of positive elements in the weight function, and the numbers of negative eigenvalues are equal to the numbers of negative elements in the weight function. Furthermore, these eigenvalues, including the eigenvalues of Neumann problem, satisfy the certain order relation.

However, all of above papers provide no information about the sign of the eigenfunctions of (4) and (5). In particular, they give no information about the sign of the eigenfunctions corresponding to two simple eigenvalues $\lambda_{1}^{+}$ and $\lambda_{1}^{-}$.

It is the purpose of this paper to show the existence of the principal eigenvalues and determine the sign of the corresponding eigenfunctions for linear periodic eigenvalue problem

$$
\begin{aligned}
-\Delta^{2} u(j-1)+q(j) u(j) & =\lambda g(j) u(j), \quad j \in \mathbb{T}, \\
u(0) & =u(T), \\
u(1) & =u(T+1),
\end{aligned}
$$

where $q(j) \geq 0$ and $q(j) \not \equiv 0$ in $\mathbb{T}$, and the weight function $g$ changes its sign in $\mathbb{T}$. Our approach is motivated by Brown and Lin [9] and Smoller [10], where the infimum of Rayleigh quotient is used to characterize the principal eigenvalues for diverse linear eigenvalue problems of elliptic equations with infinite weight.

For the other recent results on the spectrum structure of discrete linear eigenvalue problems with one-sign weight, see Sun and Shi [11], Shi and Chen [12], Jirari [13], Bohner [14], and Agarwal et al. [15] and the references therein.

The rest of the paper is organized as follows. In Section 2, we show the existence of the principal eigenvalues of (7) and determine the sign of the corresponding eigenfunctions. In Section 3, we apply our spectrum theory and the wellknown Rabinowitz bifurcation theorem to show the existence of positive solutions for nonlinear discrete periodic boundary value problem

$$
\begin{aligned}
-\Delta^{2} u(j-1)+q(j) u(j) & =f(j, u(j)), \quad j \in \mathbb{T}, \\
u(0) & =u(T), \\
u(1) & =u(T+1),
\end{aligned}
$$

under $\lim _{s \rightarrow 0}(f(j, s) / s)=g(j), j \in \mathbb{T}$, where $q(j) \geq 0$ and $q(j) \not \equiv 0$ in $\mathbb{T}, f: \mathbb{T} \times[0, \infty) \rightarrow \mathbb{R}$ is a continuous function, and $g: \mathbb{T} \rightarrow \mathbb{R}$ changes its sign.

\section{Existence of Principal Eigenvalues}

In this section, we consider the linear eigenvalue problem (7) under the following assumptions:

$(\mathrm{H} 0) q: \mathbb{T} \rightarrow[0, \infty)$, and $q\left(j_{0}\right)>0$ for some $j_{0} \in \mathbb{T}$.

(H1) $g: \mathbb{T} \rightarrow \mathbb{R}$, and there exists a proper subset, $\mathbb{\mathbb { T }}^{+}$, of $\mathbb{}$, such that $g(j)>0$ for $j \in \mathbb{T}^{+}$and $g(j)<0$ for $j \in \mathbb{T} \backslash \mathbb{T}^{+}$. Let $n$ be the number of elements in $\mathbb{T}^{+}$. Then $T-n$ is the number of elements in $\mathbb{T} \backslash \mathbb{T}^{+}$.
The difference operator is $L_{0}$, defined by

$$
\begin{aligned}
& L_{0} u(j)=-\Delta^{2} u(j-1)+q(j) u(j) \\
& \qquad \text { for } u \in \mathscr{D}, j \in \mathbb{T},
\end{aligned}
$$

where

$$
\begin{aligned}
\mathscr{D} & =\{(u(0), u(1), \ldots, u(T), u(T+1)): u(0) \\
& =u(T), u(1)=u(T+1)\} .
\end{aligned}
$$

Define a linear operator $T_{0}: \mathscr{D} \rightarrow \mathscr{D}$ by

$$
\begin{aligned}
& T_{0} u(j) \\
& = \begin{cases}-\Delta^{2} u(j-1)+q(j) u(j)-\lambda g(j) u(j), & \text { for } j \in \mathbb{T}, \\
T_{0} u(T), & \text { for } j=0, \\
T_{0} u(1), & \text { for } j=T+1 .\end{cases}
\end{aligned}
$$

Then, it is easy to see that $T_{0}: \mathscr{D} \rightarrow \mathscr{D}$ is isomorphism. Moreover, $T_{0}: \mathscr{D} \rightarrow \mathscr{D}$ is a self-adjoint operator whose spectrum consists only of the real eigenvalues.

Let us denote the norm and the inner product of $\mathscr{D}$ by

$$
\begin{aligned}
\|v\|^{2}= & \sum_{j=1}^{T} v^{2}(j) \\
& \text { for } v=(v(0), v(1), \ldots, v(T), v(T+1)) \in \mathscr{D}, \\
\langle u, v\rangle= & \sum_{j=1}^{T} v(j) u(j), \quad u, v \in \mathscr{D},
\end{aligned}
$$

respectively. For $v \in \mathscr{D}$, we have from [16, Lemma 2.1] that

$$
\left\langle-\Delta^{2} v(j-1), v(j)\right\rangle=\langle\Delta v(j), \Delta v(j)\rangle .
$$

Thus, we may define a functional

$$
\begin{aligned}
Q_{\lambda}(v)= & \langle\Delta v(j), \Delta v(j)\rangle+\langle q(j) v(j), v(j)\rangle \\
& -\lambda \sum_{j=1}^{T} g(j) v^{2}(j) .
\end{aligned}
$$

To study the principal eigenvalues of (7), we need the following preliminary lemmas.

Lemma 1. Let (HO) and (H1) hold. Assume there is a nonnegative eigenfunction corresponding to an eigenvalue $\lambda$ of (7). Then, $Q_{\lambda}(v) \geq 0$ for all $v \in \mathscr{D}$.

Proof. Suppose that $u$ is a nonnegative eigenfunction corresponding to the eigenvalue $\lambda$. Then, $u$ is a nonnegative eigenfunction corresponding to the eigenvalue $r=k$ of

$$
\begin{aligned}
& -\Delta^{2} u(j-1)+q(j) u(j)-\lambda g(j) u(j)+k u(j) \\
& \quad=r u(j), \quad j \in \mathbb{T}, \\
& u(0)=u(T) \\
& u(1)=u(T+1) .
\end{aligned}
$$


Let

$$
\begin{array}{r}
T_{0} u(j)=-\Delta^{2} u(j-1)+q(j) u(j)-\lambda g(j) u(j), \\
u \in \mathscr{D},
\end{array}
$$

and $T_{k} u(j):=\left(T_{0} u\right)(j)+k u(j)$. Then, $T_{k}^{-1}: \mathscr{D} \rightarrow \mathscr{D}$ is strongly positive if $k$ is large enough. Clearly, $r$ and $\psi$ are the eigenvalue and eigenfunction of $T_{k}$ if and only if $\mu=r-k$ and $\psi$ are the corresponding eigenvalue and eigenfunction of the problem

$$
\begin{aligned}
& -\Delta^{2} u(j-1)+q(j) u(j)-\lambda g(j) u(j)=\mu u(j), \\
& j \in \mathbb{T}, \\
& u(0)=u(T), \\
& u(1)=u(T+1) \text {. }
\end{aligned}
$$

Since $T_{k}: \mathscr{D} \rightarrow \mathscr{D}$ is a self-adjoint operator, its spectrum contains only real eigenvalues:

$$
\eta_{1} \leq \eta_{2} \leq \cdots \leq \eta_{T} .
$$

Moreover, by the well-known Krein-Rutman theorem [17, Theorem 19.2], $\eta_{1}>0$ is simple, and its corresponding eigenfunction $\psi_{1}$ is of one sign.

So, the eigenvalue $\mu_{1}\left(=\eta_{1}-k\right)$ is simple and the corresponding eigenfunction $\psi_{1}$ does not change sign in $\mathbb{T}$. Notice $k$ and $u$ are eigenvalue and the corresponding eigenfunction of (16) and (17), respectively, and $u$ is not orthogonal to $\psi_{1}$. This together with the fact that eigenfunctions corresponding to distinct eigenvalues are orthogonal implies that $u$ must be an eigenfunction of $T_{k}$ corresponding to $\eta_{1}\left(=\mu_{1}+k\right)$. Hence, $\mu_{1}=0$. By the spectral theorem

$$
\left\langle T_{0} v, v\right\rangle \geq \mu_{1}\langle v, v\rangle=0 \quad \forall v \in \mathscr{D} ;
$$

that is, $Q_{\lambda}(v) \geq 0$ for all $v \in \mathscr{D}$.

Lemma 2. Let (HO) and (H1) hold. Let

$$
\begin{aligned}
K(v) & =\frac{\sum_{j=1}^{T}\left[|\Delta v(j)|^{2}+q(j) v^{2}(j)\right]}{\sum_{j=1}^{T} g(j) v^{2}(j)}, \\
\lambda_{1}^{+} & =\inf \left\{K(v): v \in \mathscr{D}, \sum_{j=1}^{T} g(j) v^{2}(j)>0\right\} .
\end{aligned}
$$

Then, $\lambda_{1}^{+} \in(0, \infty)$.

Proof. By the spectral theorem, $\left\langle L_{0} v, v\right\rangle \geq \gamma_{1}\langle v, v\rangle$ for all $v \in \mathscr{D}$, where $\gamma_{1}$ is the first eigenvalue of $L_{0}$. Note that (H0) implies that

$$
\gamma_{1}>0
$$

Hence, if $v \in \mathscr{D}$ with $\sum_{j=1}^{T} g(j) v^{2}(j)>0$, then

$$
\begin{aligned}
K(v) & =\frac{\left\langle L_{0} v, v\right\rangle}{\sum_{j=1}^{T} g(j) v^{2}(j)} \geq \frac{\gamma_{1}\langle v, v\rangle}{\sum_{j=1}^{T} g(j) v^{2}(j)} \\
& \geq \frac{\gamma_{1}}{\sup _{j \in T}|g(j)|}>0 .
\end{aligned}
$$

Hence

$$
\lambda_{1}^{+} \geq \frac{\gamma_{1}}{\sup _{j \in \mathbb{T}}|g(j)|}>0 .
$$

Lemma 3. If $\lambda>\lambda_{1}^{+}$, then $\lambda$ is not an eigenvalue of (7) possessing a nonnegative eigenfunction.

Proof. If $\lambda>\lambda_{1}^{+}$, there exists $v \in \mathscr{D}$ such that $\sum_{j=1}^{T} g(j) v^{2}(j)>$ 0 and $K(v)<\lambda$; that is,

$$
\frac{\sum_{j=1}^{T}\left[|\Delta v(j)|^{2}+q(j) v^{2}(j)\right]}{\sum_{j=1}^{T} g(j) v^{2}(j)}<\lambda
$$

and so $Q_{\lambda}(v)<0$. The required result is now an immediate consequence of Lemma 1.

Lemma 4. Assume (H1) and $0<\lambda<\lambda_{1}^{+}$. Then, there exists $a>0$ (a depends on $\lambda$ ) such that $Q_{\lambda}(v) \geq a\|v\|^{2}$ for all $v \in \mathscr{D}$.

Proof. Let $\lambda=(1-s) \lambda_{1}^{+}$, where $0<s<1$.

We claim that

$$
Q_{\lambda}(v) \geq s \gamma_{1}\|v\|^{2} .
$$

In fact, for $v \in \mathscr{D}$, we have from the fact that

$$
\begin{aligned}
Q_{\lambda_{1}^{+}}(v)= & \sum_{j=1}^{T}|\Delta v(j)|^{2}+\sum_{j=1}^{T} q(j) v^{2}(j) \\
& -\sum_{j=1}^{T} \lambda_{1}^{+} g(j) v^{2}(j) \geq 0, \quad v \in \mathscr{D}
\end{aligned}
$$

that

$$
\begin{aligned}
& Q_{\lambda}(v) \\
& =\sum_{j=1}^{T}|\Delta v(j)|^{2}+\sum_{j=1}^{T} q(j) v^{2}(j)-\sum_{j=1}^{T} \lambda g(j) v^{2}(j) \\
& =\frac{\lambda}{\lambda_{1}^{+}} Q_{\lambda_{1}^{+}}(v) \\
& +\left(1-\frac{\lambda}{\lambda_{1}^{+}}\right)\left[\sum_{j=1}^{T}|\Delta v(j)|^{2}+\sum_{j=1}^{T} q(j) v^{2}(j)\right] \\
& \geq s \gamma_{1} \sum_{j=1}^{T}|v(j)|^{2} \text {. }
\end{aligned}
$$

Lemma 5. Let (HO) and (H1) hold. Let

$$
\lambda_{1}^{-}=\sup \left\{K(v): v \in \mathscr{D}, \sum_{j=1}^{T} g(j) v^{2}(j)<0\right\} .
$$

Then, $\lambda_{1}^{-} \in(-\infty, 0)$. 
Proof. If $v \in \mathscr{D}$ with $\sum_{j=1}^{T} g(j) v^{2}(j)<0$, then it follows from the fact that

$$
\left\langle L_{0} v, v\right\rangle \geq \gamma_{1}\langle v, v\rangle \quad v \in D
$$

that

$$
\begin{aligned}
K(v) & =\frac{\left\langle L_{0} v, v\right\rangle}{\sum_{j=1}^{T} g(j) v^{2}(j)} \leq \frac{\gamma_{1}\langle v, v\rangle}{\sum_{j=1}^{T} g(j) v^{2}(j)} \\
& \leq-\frac{\gamma_{1}}{\sup _{j \in T}|g(j)|}<0 .
\end{aligned}
$$

Hence,

$$
\lambda_{1}^{-} \leq-\frac{\gamma_{1}}{\sup _{j \in \mathbb{T}}|g(j)|}<0
$$

Lemma 6. If $\lambda<\lambda_{1}^{-}$, then $\lambda$ is not an eigenvalue of (7) possessing a nonnegative eigenfunction.

Proof. If $\lambda<\lambda_{1}^{-}$, then there exists $v \in \mathscr{D}$ such that $\sum_{j=1}^{T} g(j) v^{2}(j)<0$ and $K(v)>\lambda$; that is,

$$
\frac{\sum_{j=1}^{T}\left[|\Delta v(j)|^{2}+q(j) v^{2}(j)\right]}{\sum_{j=1}^{T} g(j) v^{2}(j)}>\lambda
$$

and so $Q_{\lambda}(v)<0$. The required result is now an immediate consequence of Lemma 1.

Lemma 7. Assume (H1) and $\lambda_{1}^{-}<\lambda<0$. Then, there exists $a>0$ (a depends on $\lambda$ ) such that $Q_{\lambda}(v) \geq a\|v\|^{2}$ for all $v \in \mathscr{D}$.

Proof. Let $\lambda=(1-s) \lambda_{1}^{-}$, where $0<s<1$.

For $v \in \mathscr{D}$, we have from

$$
\begin{aligned}
Q_{\lambda_{1}^{-}}(v)= & \sum_{j=1}^{T}|\Delta v(j)|^{2}+\sum_{j=1}^{T} q(j) v^{2}(j) \\
& -\sum_{j=1}^{T} \lambda_{1}^{-} g(j) v^{2}(j) \geq 0, \quad v \in \mathscr{D}
\end{aligned}
$$

that

$$
\begin{aligned}
Q_{\lambda}(v) & \\
= & \sum_{j=1}^{T}|\Delta v(j)|^{2}+\sum_{j=1}^{T} q(j) v^{2}(j)-\sum_{j=1}^{T} \lambda g(j) v^{2}(j) \\
= & \frac{\lambda}{\lambda_{1}^{-}} Q_{\lambda_{1}^{-}}(v) \\
& +\left(1-\frac{\lambda}{\lambda_{1}^{-}}\right)\left[\sum_{j=1}^{T}|\Delta v(j)|^{2}+\sum_{j=1}^{T} q(j) v^{2}(j)\right] \\
\geq & s \gamma_{1} \sum_{j=1}^{T}|v(j)|^{2} .
\end{aligned}
$$

Theorem 8. If (HO) and (H1) hold, then (7) has exactly two principal eigenvalues $\lambda_{1}^{-}$and $\lambda_{1}^{+}$, such that

(1) $\lambda_{1}^{-}<0<\lambda_{1}^{+}$;

(2) the algebraic multiplicity of $\lambda_{1}^{-}$and $\lambda_{1}^{+}$is 1 ;

(3) the eigenfunctions $\varphi_{1}^{-}$and $\varphi_{1}^{+}$corresponding to the eigenvalues $\lambda_{1}^{-}$and $\lambda_{1}^{+}$are of one sign.

Proof. Consider the linear eigenvalue problem

$$
-\Delta^{2} u(j-1)+q(j) u(j)-\lambda_{1}^{+} g(j) u(j)=\mu u(j),
$$

$$
\begin{aligned}
& u(0)=u(T), \\
& u(1)=u(T+1) .
\end{aligned}
$$

It is easy to see that $\lambda_{1}^{+}$is an eigenvalue for (7) with corresponding eigenfunction $w$ if and only if 0 is an eigenvalue of $T_{0}$, and, accordingly, 0 is an eigenvalue of (38) with corresponding eigenfunction $w$. The least eigenvalue of $T_{0}$ is given by

$$
\begin{aligned}
\alpha_{1} & =\inf \left\{\sum_{j=1}^{T}|\Delta v(j)|^{2}+\sum_{j=1}^{T} q(j) v^{2}(j)\right. \\
& \left.-\sum_{j=1}^{T} \lambda_{1}^{+} g(j) v^{2}(j): v \in \mathscr{D}\right\}=\inf \left\{Q_{\lambda_{1}^{+}}(v): v\right. \\
& \in \mathscr{D}\} .
\end{aligned}
$$

Since $Q_{\lambda_{1}^{+}}(v) \geq 0$ for all $v \in \mathscr{D}, \alpha_{1} \geq 0$. Because of how we defined $\lambda_{1}^{+}$, there exists a sequence $\left\{v_{n}\right\} \subset \mathscr{D}$ such that $\sum_{j=1}^{T} g(j) v_{n}^{2}(j)=1$ and

$$
\begin{aligned}
\lim _{n \rightarrow \infty} K\left(v_{n}\right) & =\lim _{n \rightarrow \infty}\left[\sum_{j=1}^{T}\left|\Delta v_{n}(j)\right|^{2}+\sum_{j=1}^{T} q(j) v_{n}^{2}(j)\right] \\
& =\lambda_{1}^{+} .
\end{aligned}
$$

Therefore,

$$
\lim _{n \rightarrow \infty} Q_{\lambda_{1}^{+}}\left(v_{n}\right)=0
$$

and so $\alpha_{1} \leq 0$. Hence, $\alpha_{1}=0$ is the least eigenvalue of (38) and so $\alpha_{1}$ is simple and the corresponding eigenfunction can be chosen to be positive on $\mathbb{T}$.

Using the same method, with obvious changes, we may prove the algebraic multiplicity of $\lambda_{1}^{-}$is 1 and the eigenfunction $\varphi_{1}^{-}$corresponding to the eigenvalue $\lambda_{1}^{-}$is of one sign.

\section{Existence of Positive Solutions}

As an application, we consider the existence of positive solutions of the discrete nonlinear problem (8) and (9).

In this section, we assume that

(H2) $\lim _{s \rightarrow 0}(f(j, s) / s)=g(j)$ for all $j \in \mathbb{T}$; 
(H3) $\lim _{s \rightarrow \infty}(f(j, s) / s)=m(j)$ for all $j \in \mathbb{T}$, where $m$ : $\mathbb{T} \rightarrow[0, \infty)$ and $m(j)>0$ for all $j \in \mathbb{T}$.

Let $\lambda_{1}(m)$ be the principal eigenvalue of

$$
\begin{aligned}
-\Delta^{2} u(j-1)+q(j) u(j) & =\lambda m(j) u(j), \quad j \in \mathbb{T}, \\
u(0) & =u(T), \\
u(1) & =u(T+1),
\end{aligned}
$$

and let $\phi$ be an eigenfunction corresponding to $\lambda_{1}(m)$.

Theorem 9. Assume that (HO)-(H3) hold. Then

(1) (8) and (9) have at least one positive solution for $\lambda_{1}^{+}<$ $1<\lambda_{1}(m)$;

(2) (8) and (9) have at least one positive solution for $\lambda_{1}(m)<1<\lambda_{1}^{+}$.

Proof.

Step 1. A bifurcation result.

We extend the function $f$ to a continuous function $\tilde{f}$ defined on $\mathbb{T} \times \mathbb{R}$ by setting, for $j \in \mathbb{T}$,

$$
\tilde{f}(j, s)= \begin{cases}f(j, s) & \text { if } s \geq 0, \\ -f(j,-s) & \text { if } s<0 .\end{cases}
$$

Obviously, within the context of positive solutions, problem (8) and (9) is equivalent to the same problem with $f$ replaced by $\tilde{f}$. Furthermore, $\tilde{f}(j, s)$ is an odd function for $j \in \mathbb{T}$. In the sequel of the proof, we shall replace $f$ with $\widetilde{f}$. However, for the sake of simplicity, the modified function $\widetilde{f}$ will still be denoted by $f$.

Recall that

$$
\begin{aligned}
& L_{0} u(j)=-\Delta^{2} u(j-1)+q(j) u(j) \\
& \qquad \text { for } u \in \mathscr{D}, j \in \mathbb{T},
\end{aligned}
$$

where

$$
\begin{aligned}
\mathscr{D} & =\{(u(0), u(1), \ldots, u(T), u(T+1)): u(0) \\
& =u(T), u(1)=u(T+1)\} .
\end{aligned}
$$

Let $\xi, \zeta \in C(\mathbb{T} \times \mathbb{R}, \mathbb{R})$ be such that

$$
\begin{aligned}
& f(j, u)=g(j) u+\xi(j, u), \\
& f(j, u)=m(j) u+\zeta(j, u)
\end{aligned}
$$

$\forall j \in \mathbb{T}$,

where

$$
\begin{aligned}
\lim _{u \rightarrow 0} \frac{\xi(j, u)}{u} & =0, \\
\lim _{u \rightarrow \infty} \frac{\zeta(j, u)}{u} & =0
\end{aligned}
$$

Let us consider

$$
L_{0} u-\lambda g(j) u=\lambda \xi(j, u)
$$

as a bifurcation problem from the trivial solution $u \equiv 0$. A solution of (48) is a pair $(\lambda, u) \in \mathbb{R} \times \mathscr{D}$ which satisfies (48). It is easy to see that any solution of $(48)$ of the form $(1, u)$ yields a solution $u$ of (8) and (9).

Equation (48) can be converted into the equivalent equation

$$
u(j)=\lambda L_{0}^{-1}[g(\cdot) u(\cdot)](j)+\lambda L_{0}^{-1}[\xi(\cdot, u(\cdot))](j) .
$$

Further, we note that $\lim _{u \rightarrow 0}\left(L_{0}^{-1}[\xi(\cdot, u(\cdot))] /\|u\|\right)=0$ in $\mathscr{D}$, since

$$
\begin{aligned}
& \left\|L_{0}^{-1}[\xi(\cdot, u(\cdot))]\right\| \\
& \quad=\left[\sum_{j=1}^{T}\left(\sum_{t=1}^{T} G(t, j) \xi(t, u(t))\right)^{2}\right]^{1 / 2} \\
& \quad \leq C \cdot\|\xi(\cdot, u(\cdot))\|,
\end{aligned}
$$

where $C=\sqrt{T} \max _{j \in \mathbb{\mathbb { N }}} \sum_{t=1}^{T} G(t, j)$ and $G(t, j)$ is given by [18, Theorem 2.1]. We denote (49) by

$$
u=\lambda \mathscr{L} u+\mathscr{H}(\lambda, u) .
$$

Clearly, $\mathscr{L}$ and $\mathscr{H}: \mathbb{R} \times \mathscr{D} \rightarrow \mathscr{D}$ are completely continuous and

$$
\lim _{u \rightarrow 0} \frac{\|\mathscr{H}(\lambda, u)\|}{\|u\|}=0
$$

with respect to $\lambda$ varying in bounded intervals. Notice that if $\lambda$ is the eigenvalue of $L_{0}$, then it also is the characteristic value of $\mathscr{L}$.

We say that a solution $(\lambda, u) \in \mathbb{R} \times \mathscr{D}$ of (51) is nontrivial if there exists $j_{0} \in \mathbb{T}$ such that $u\left(j_{0}\right) \neq 0$. Denote by $\delta$ the closure in $\mathbb{R} \times \mathscr{D}$ of the set of all nontrivial solutions $(\lambda, u)$ of (51) with $\lambda>0$.

Theorem 1.3 in [19] yields the existence of a maximal closed connected set $\mathscr{C}$ in $\mathcal{S}$ such that $\left(\lambda_{1}^{+}, 0\right) \in \mathscr{C}$ and at least one of the following conditions holds:

(i) $\mathscr{C}$ is unbounded in $\mathbb{R} \times \mathscr{D}$.

(ii) There exists a characteristic value of $\mathscr{L}$, with $\hat{\lambda} \neq \lambda_{1}^{+}$, such that $(\hat{\lambda}, 0) \in \mathscr{C}$.

Step 2. In what follows, we prove several properties which will eventually lead to the fact that condition (ii) above does not hold.

Claim 1. Suppose $(\tilde{\lambda}, 0) \in \mathcal{S}$, and then $\tilde{\lambda}$ is a characteristic value of $\mathscr{L}$. Let $\left\{\left(\lambda^{[k]}, u^{[k]}\right)\right\}$ be a sequence of nontrivial solutions of (51), converging to $(\tilde{\lambda}, 0)$ in $\mathbb{R} \times \mathscr{D}$.

Setting, for all $k, v^{[k]}=u^{[k]} /\left\|u^{[k]}\right\|$, we have

$$
v^{[k]}=\lambda^{[k]} \mathscr{L}\left(v^{[k]}\right)+\frac{\mathscr{H}\left(\lambda^{[k]}, u^{[k]}\right)}{\left\|u^{[k]}\right\|} .
$$


As $\left\{v^{[k]}\right\}$ is bounded in $\mathscr{D}$ and $\mathscr{L}$ is completely continuous, there exist $w \in \mathscr{D}$ and a subsequence of $\left\{v^{[k]}\right\}$, which we denote in the same way, such that

$$
\lim _{k \rightarrow+\infty} \mathscr{L}\left(v^{[k]}\right)=w \quad \text { in } \mathscr{D} \text {. }
$$

Hence, we conclude by (53) that

$$
\lim _{k \rightarrow+\infty} v^{[k]}=\tilde{\lambda} w \text { in } \mathscr{D} .
$$

Therefore, we have

$$
w=\tilde{\lambda} \mathscr{L}(w)
$$

with $\|\widetilde{\lambda} w\|=1$ and in particular $w \neq 0$. Accordingly, $\tilde{\lambda}$ is a characteristic value of $\mathscr{L}$.

Claim 2. There exists $\epsilon>0$ such that $\mathcal{S} \subset[\epsilon,+\infty) \times \mathscr{D}$.

By contradiction, we can suppose that there exists a sequence $\left\{\left(\lambda^{[k]}, u^{[k]}\right)\right\}$ of nontrivial solutions of (51), converging in $\mathbb{R} \times \mathscr{D}$ to some $(0, u) \in \mathbb{R} \times \mathscr{D}$. Arguing as in the proof of Claim 1, we set $v^{[k]}=u^{[k]} /\left\|u^{[k]}\right\|$, and we have

$$
v^{[k]}=\lambda^{[k]} \mathscr{L}\left(v^{[k]}\right)+\frac{\mathscr{H}\left(\lambda^{[k]}, u^{[k]}\right)}{\left\|u^{[k]}\right\|}
$$

and conclude that, possibly passing to a subsequence, $\lim _{k \rightarrow \infty} v^{[k]}=0$ in $\mathscr{D}$, which contradicts $\left\|v^{[k]}\right\|=1$.

Claim 3. $(\lambda, u) \in \mathscr{C}$ if and only if $(\lambda,-u) \in \mathscr{C}$.

This follows from the fact that $f$, and hence $\mathscr{H}$, is odd with respect to the second variable.

In the sequel, we denote by $P$ the positive cone in $\mathscr{D}$; that is,

$$
P=\{u \in \mathscr{D}: u \geq 0\},
$$

and we denote by int $P$ its interior and by $\partial P$ its boundary.

Claim 4. There exists a neighborhood $U$ of $\left(\lambda_{1}^{+}, 0\right)$ in $\mathbb{R} \times \mathscr{D}$ such that, for all $(\lambda, u) \in \mathscr{C} \cap U$, either $(\lambda, u)=\left(\lambda_{1}^{+}, 0\right)$, or $u \in$ int $P$, or $-u \in$ int $P$.

It is an immediate consequence of the fact that $\varphi_{1}^{+}(j)>0$ for all $j \in \mathbb{T}$ and the well-known Crandall-Rabinowitz local bifurcation theorem; see Crandall and Rabinowitz [20] and Kielhöfer [21].

Claim 5. Assume $(\lambda, u) \in \mathscr{C}$ and $u \in \partial P$. Suppose further that $(\lambda, u)$ is the limit of a sequence $\left\{\left(\lambda^{[k]}, u^{[k]}\right)\right\}$ in $\mathscr{C}$, with $u^{[k]}>0$ for all $k$. Then, $(\lambda, u)=\left(\lambda_{1}^{+}, 0\right)$.

We first show that $u=0$.

Suppose, by contradiction, that $u>0$. Then, we can take $c>0$ such that

$$
\lambda\left(g(j)+\frac{\xi(j, u)}{u}\right)+c \geq 1, \quad j \in \mathbb{T} .
$$

Hence, we get

$$
\begin{aligned}
- & \Delta^{2} u(j-1)+q(j) u(j)+c u(j) \\
& =\left(\lambda\left(g(j)+\frac{\xi(j, u)}{u}\right)+c\right) u(j), \quad j \in \mathbb{T} .
\end{aligned}
$$

As $u \in P$ satisfies

$$
\begin{gathered}
\left(\lambda\left(g(j)+\frac{\xi(j, u)}{u}\right)+c\right) u(j) \geq 0, \quad j \in \mathbb{T}, \\
\left(\lambda\left(g\left(j_{0}\right)+\frac{\xi\left(j_{0}, u\right)}{u}\right)+c\right) u\left(j_{0}\right)>0,
\end{gathered}
$$

for some $j_{0} \in \mathbb{T}$, let $G(t, s)$ be the Green function for the linear boundary value problem

$$
\begin{aligned}
-\Delta^{2} u(j-1)+q(j) u(j)+c u(j) & =0, \\
u(0) & =u(T), \\
u(1) & =u(T+1) .
\end{aligned}
$$

Since the Green function $G(j, s)$ for $(62)$ satisfies $G(j, s)>0$ on $0 \leq j, s \leq T$ (see [18, Theorems 2.1 and 2.2] for details), this yields $u(j)>0$ for $j \in \mathbb{T}$, contradicting $u \in \partial P$. Therefore, we conclude that $u=0$. We next show that $\lambda=\lambda_{1}^{+}$. By Claim 1 , $\lambda$ is a characteristic value of $\mathscr{L}$. Setting $v^{[k]}=u^{[k]} /\left\|u^{[k]}\right\|$ and arguing as in the proof of Claim 1, we conclude that, possibly passing to a subsequence,

$$
\lim _{k \rightarrow \infty} \mathscr{L}\left(v^{[k]}\right)=w
$$

in $\mathscr{D}$, where $w$ is an eigenfunction of (7) associated with $\lambda$. Since $w>0$, we conclude that $\lambda=\lambda_{1}^{+}$.

Claim 6. For all $(\lambda, u) \in \mathscr{C}$, either $u \in \operatorname{int} P$, or $-u \in \operatorname{int} P$, or $(\lambda, u)=\left(\lambda_{1}^{+}, 0\right)$

Set

$$
\begin{aligned}
\mathscr{E} & =\{(\lambda, u) \in \mathscr{C}: u \notin \operatorname{int} P,-u \notin \operatorname{int} P,(\lambda, u) \\
& \left.\neq\left(\lambda_{1}^{+}, 0\right)\right\} .
\end{aligned}
$$

By Claim 4,

$$
\mathscr{E}=\{(\lambda, u) \in(\mathscr{C} \backslash U): u \notin \operatorname{int} P,-u \notin \operatorname{int} P\}
$$

and, subsequently, $\mathscr{E}$ is a closed subset of $\mathscr{C}$.

Let us verify that $\mathscr{E}$ is open in $\mathscr{C}$. Suppose this is not the case. Then, there exist $(\lambda, u) \in \mathscr{E}$ and a sequence $\left\{\left(\lambda^{[k]}, u^{[k]}\right)\right\}$ in $\mathscr{C} / \mathscr{E}$ converging to $(\lambda, u)$. We may assume that $u^{[k]} \epsilon$ int $P$ for all $k$; hence, by Claim 5 , we obtain $(\lambda, u)=\left(\lambda_{1}^{+}, 0\right)$, contradicting the fact that $(\lambda, u) \in \mathscr{E}$. As $\mathscr{C}$ is connected and $\left(\lambda_{1}^{+}, 0\right) \in \mathscr{C} \backslash \mathscr{E}$, we conclude that $\mathscr{E}=\emptyset$.

By Claim 6 we have that if $(\hat{\lambda}, 0) \in \mathscr{C}$, then $\hat{\lambda}=\lambda_{1}^{+}$ and hence condition (ii) above does not hold. Consequently, condition (i) is valid.

Step 3. Next, let us show that $\mathscr{C}$ joins $\left(\lambda_{1}^{+}, 0\right)$ to $\left(\lambda_{1}(m), \infty\right)$. Let $\left\{\left(\mu^{[k]}, y^{[k]}\right)\right\} \in \mathscr{C}$ satisfy

$$
\mu^{[k]}+\left\|y^{[k]}\right\| \longrightarrow \infty .
$$

We note that $\mu^{[k]}>0$ for all $k \in \mathbb{N}$, since $(0,0)$ is the only solution of (51) for $\lambda=0$ and $\mathscr{C} \cap(\{0\} \times \mathscr{D})=\emptyset$. 
We first show that there exists a constant $M>0$, such that

$$
\mu^{[k]} \in(0, M] \quad \forall k \in \mathbb{N} .
$$

Suppose (67) does not hold, then choosing a subsequence and relabelling if necessary, it follows that

$$
\lim _{k \rightarrow \infty} \mu^{[k]}=\infty
$$

We divide the equation

$$
y^{[k]}(j)=\mu^{[k]} \sum_{s=1}^{T} G(j, s) f\left(s, y^{[k]}(s)\right)
$$

by $\left\|y^{[k]}\right\|$ and set $v^{[k]}=y^{[k]} /\left\|y^{[k]}\right\|$, and we have

$$
v^{[k]}(j)=\mu^{[k]} \sum_{s=1}^{T} G(j, s) \frac{f\left(s, y^{[k]}(s)\right)}{y^{[k]}(s)} v^{[k]}(s)
$$

for all $j \in \mathbb{T}$. Since $\left\{v^{[k]}\right\}$ is bounded in $\mathscr{D}$, after taking a subsequence and relabelling if necessary, we have that $v^{[k]} \rightarrow$ $\bar{v}$ for all $k \in \mathbb{N}$, where $\bar{v} \in \mathscr{D}$ with $\|\bar{v}\|=1$.

It follows from (66) and (68) that either

$$
\begin{aligned}
\left\|y^{[k]}\right\| & \in(0, \infty) \\
\text { or }\left\|y^{[k]}\right\| & \longrightarrow \infty .
\end{aligned}
$$

Combining this fact with (H3) and

$$
y^{[k]}(j) \geq \gamma\left\|y^{[k]}\right\| \quad \forall j \in \mathbb{T},
$$

where $\gamma>0$ with $G(j, s) \geq \gamma G(s, s)$, we have

$$
\bar{v}(j)=\mu^{[k]} \sum_{s=1}^{T} G(j, s) m(s) \bar{v}(s),
$$

this is a contradiction, and therefore (67) holds.

According to (67), we have

$$
\begin{aligned}
\left\|y^{[k]}\right\| & \longrightarrow \infty, \\
k & \longrightarrow \infty .
\end{aligned}
$$

Since $\left(\mu^{[k]}, y^{[k]}\right)$ solve

$$
L_{0} y^{[k]}-\mu^{[k]} m(j) y^{[k]}=\mu^{[k]} \zeta\left(j, y^{[k]}\right),
$$

we divide (75) by $\left\|y^{[k]}\right\|$ and set $\bar{y}^{k}=y^{[k]} /\left\|y^{[k]}\right\|$. Then, we get

$$
L_{0} \bar{y}^{[k]}=\mu^{[k]} m(j) \bar{y}^{k}+\mu^{[k]} \frac{\xi\left(j, y^{[k]}(j)\right)}{\left\|y^{[k]}\right\|} .
$$

Since $\left\{\bar{y}^{[k]}\right\}$ is bounded in $\mathscr{D}$, after taking a subsequence and relabelling if necessary, we have that $\bar{y}^{[k]} \rightarrow \bar{y}$ for some $\bar{y} \in \mathscr{D}$ with $\|\bar{y}\|=1$. Moreover, by (72) and (74), we can show that

$$
\lim _{k \rightarrow \infty} \frac{\xi\left(j, y^{[k]}(j)\right)}{\left\|y^{[k]}\right\|}=\lim _{k \rightarrow \infty} \frac{\xi\left(j, y^{[k]}(j)\right)}{y^{[k]}(j)} \cdot \bar{y}^{[k]}=0
$$

Therefore,

$$
L_{0} \bar{y}=\bar{\mu} m(j) \bar{y}
$$

where $\bar{\mu}=\lim _{k \rightarrow \infty} \mu^{[k]}$, again choosing a subsequence and relabelling if necessary.

We claim that

$$
\bar{y} \in \mathscr{C} \text {. }
$$

Suppose on the contrary that $\bar{y} \notin \mathscr{C}$. Since $\bar{y} \neq 0$ is a solution of (78) it follows that $\bar{y} \in \mathscr{C}^{k}$. By the openness of $\mathscr{D} \backslash \mathscr{C}$, we know that there exists a neighborhood $U\left(\bar{y}, \rho_{0}\right)$ such that

$$
U\left(\bar{y}, \rho_{0}\right) \subset \mathscr{D} \backslash \mathscr{C},
$$

which contradicts the facts that $\bar{y}^{[k]} \rightarrow \bar{y}$ in $\mathscr{D}$ and $\bar{y}^{[k]} \in \mathscr{C}$. Therefore, $\bar{y} \in \mathscr{C}$. Moreover, by Sturm-Liouville eigenvalue theory, $\bar{\mu}=\lambda_{1}(m)$. Therefore, $\mathscr{C}$ joins $\left(\lambda_{1}^{+}, 0\right)$ to $\left(\lambda_{1}(m), \infty\right)$.

Therefore, $\mathscr{C}$ crosses the hyperplane $\{1\} \times \mathscr{D}$ in $\mathbb{R} \times \mathscr{D}$, and, accordingly, (8) and (9) have at least one positive solution.

Remark 10. Let us consider the nonlinear problem

$$
\begin{aligned}
-\Delta^{2} u(j-1)+\frac{1}{2} u(j) & =f(j, u(j)), \quad j \in \mathbb{T}, \\
u(0) & =u(5), \\
u(1) & =u(6),
\end{aligned}
$$

where

$$
\begin{aligned}
& f(j, s)=f^{*}(s)+\beta(j, s) s, \\
& f^{*}(s)= \begin{cases}0, & s=0, \\
\frac{1}{8} s, & s \in(0,1], \\
8 s-\frac{63}{8}, & s \in(1, \infty),\end{cases} \\
& \beta(j, s)= \begin{cases}\frac{1}{4}, & j \in\{1,2,3,4\}, s \in[0, \infty), \\
-\frac{1}{4}, & j=5, s \in[0,1], \\
\frac{1}{2} s-\frac{3}{4}, & j=5, s \in(1,2), \\
\frac{1}{4}, & j=5, s \in[2, \infty) .\end{cases}
\end{aligned}
$$

Obviously,

$$
\begin{aligned}
\lim _{s \rightarrow 0} \frac{f(j, s)}{s} & =g(j), \\
\lim _{s \rightarrow+\infty} \frac{f(j, s)}{s} & =m(j)
\end{aligned}
$$

with

$$
g(j)= \begin{cases}\frac{3}{8}, & j \in\{1,2,3,4\} \\ -\frac{1}{8}, & j=5,\end{cases}
$$


and $m(j)=33 / 4$. Thus

$$
\lambda_{1}(m)=\frac{2}{33}<1
$$

On the other hand, it follows from (26) and the fact $\gamma_{1}=1 / 2$ that

$$
\lambda_{1}^{+} \geq \frac{\gamma_{1}}{\sup _{j \in \mathbb{T}}|g(j)|}=\frac{4}{3}>1
$$

Therefore, Theorem 9 (2) yields the existence of at least one positive solution of (81).

\section{Conflicts of Interest}

The authors declare that there are no conflicts of interest regarding the publication of this paper.

\section{Authors' Contributions}

$\mathrm{Ma}$ and $\mathrm{Xu}$ completed the main study together and Ma wrote the manuscript; Long was responsible for checking the proofs process and verified the calculation. Moreover, all the authors read and approved the final version of the manuscript.

\section{Acknowledgments}

This work was supported by NSFC (no. 11671322) and NSFC (no. 11361054).

\section{References}

[1] A. Constantin, "A general-weighted Sturm-Liouville problem," Annali della Scuola Normale Superiore di Pisa. Classe di Scienze. Serie IV, vol. 24, no. 4, pp. 767-782 (1998), 1997.

[2] M. S. Alber, R. Camassa, D. D. Holm, and J. E. Marsden, "The geometry of peaked solitons and billiard solutions of a class of integrable PDEs," Letters in Mathematical Physics, vol. 32, no. 2, pp. 137-151, 1994.

[3] G. Birkhoff and G.-C. Rota, Ordinary Differential Equations, John Wiley and Sons, New York, NY, USA, 1989.

[4] R. Camassa and D. D. Holm, "An integrable shallow water equation with peaked solitons," Physical Review Letters, vol. 71, no. 11, pp. 1661-1664, 1993.

[5] A. Constantin, "On the inverse spectral problem for the Camassa-Holm equation," Journal of Functional Analysis, vol. 155, no. 2, pp. 352-363, 1998.

[6] Y. Wang and Y. Shi, "Eigenvalues of second-order difference equations with periodic and antiperiodic boundary conditions," Journal of Mathematical Analysis and Applications, vol. 309, no. 1, pp. 56-69, 2005.

[7] J. Ji and B. Yang, "Eigenvalue comparisons for second order difference equations with periodic and antiperiodic boundary conditions," Applied Mathematics and Computation, vol. 27, no. 1-2, pp. 307-324, 2008.

[8] C. Gao and R. Ma, "Eigenvalues of discrete linear secondorder periodic and antiperiodic eigenvalue problems with signchanging weight," Linear Algebra and its Applications, vol. 467, pp. $40-56,2015$.
[9] K. J. Brown and S. S. Lin, "On the existence of positive eigenfunctions for an eigenvalue problem with indefinite weight function," Journal of Mathematical Analysis and Applications, vol. 75, no. 1, pp. 112-120, 1980.

[10] J. Smoller, Shock Waves and Reaction-Diffusion Equations, Springer, New York, NY, USA, 2nd edition, 1994.

[11] H. Sun and Y. Shi, "Eigenvalues of second-order difference equations with coupled boundary conditions," Linear Algebra and its Applications, vol. 414, no. 1, pp. 361-372, 2006.

[12] Y. Shi and S. Chen, "Spectral theory of second-order vector difference equations," Journal of Mathematical Analysis and Applications, vol. 239, no. 2, pp. 195-212, 1999.

[13] A. Jirari, "Second-order Sturm-Liouville difference equations and orthogonal polynomials," Memoirs of the American Mathematical Society, vol. 113, no. 542, pp. 1-138, 1995.

[14] M. Bohner, "Discrete linear Hamiltonian eigenvalue problems," Computers \& Mathematics with Applications. An International Journal, vol. 36, no. 10-12, pp. 179-192, 1998.

[15] R. P. Agarwal, M. Bohner, and P. J. Wong, "Sturm-Liouville eigenvalue problems on time scales," Applied Mathematics and Computation, vol. 99, no. 2-3, pp. 153-166, 1999.

[16] R. Ma and H. Ma, "Unbounded perturbations of nonlinear discrete periodic problem at resonance," Nonlinear Analysis, vol. 70, no. 7, pp. 2602-2613, 2009.

[17] K. Deimling, Nonlinear Functional Analysis, Springer, Berlin, Germany, 1985.

[18] F. M. Atici and G. S. Guseinov, "Positive periodic solutions for nonlinear difference equations with periodic coefficients," Journal of Mathematical Analysis and Applications, vol. 232, no. 1, pp. 166-182, 1999.

[19] P. H. Rabinowitz, "Some global results for nonlinear eigenvalue problems," Journal of Functional Analysis, vol. 7, no. 3, pp. 487$513,1971$.

[20] M. G. Crandall and P. H. Rabinowitz, "Bifurcation from simple eigenvalues," Journal of Functional Analysis, vol. 8, no. 2, pp. 321340, 1971.

[21] H. Kielhöfer, An Introduction with Applications to Partial Differential Equations, vol. 156 of Applied Mathematical Sciences, Springer, New York, NY, USA, 2nd edition, 2012. 


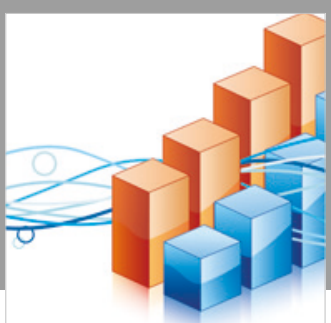

Advances in

Operations Research

\section{-n-m}
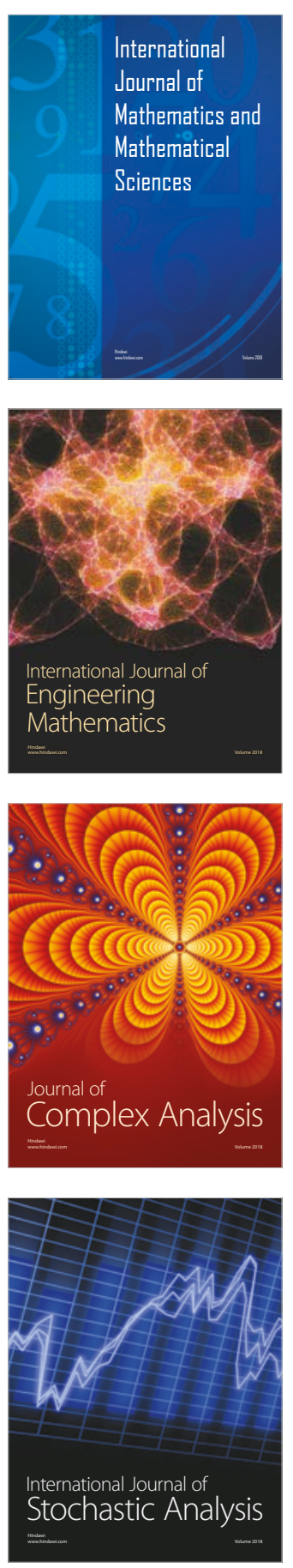
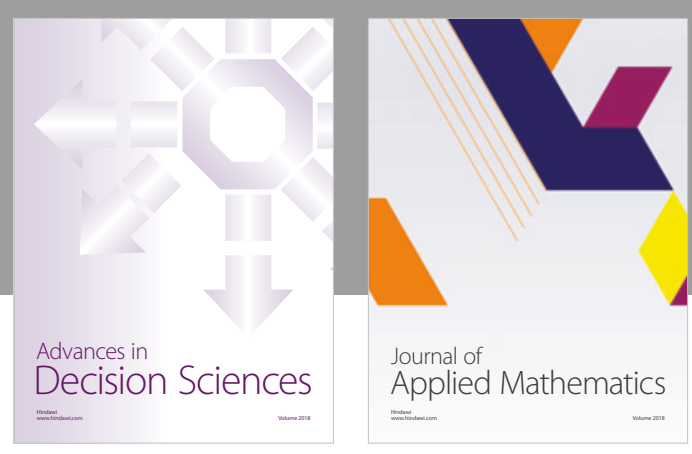

Journal of

Applied Mathematics
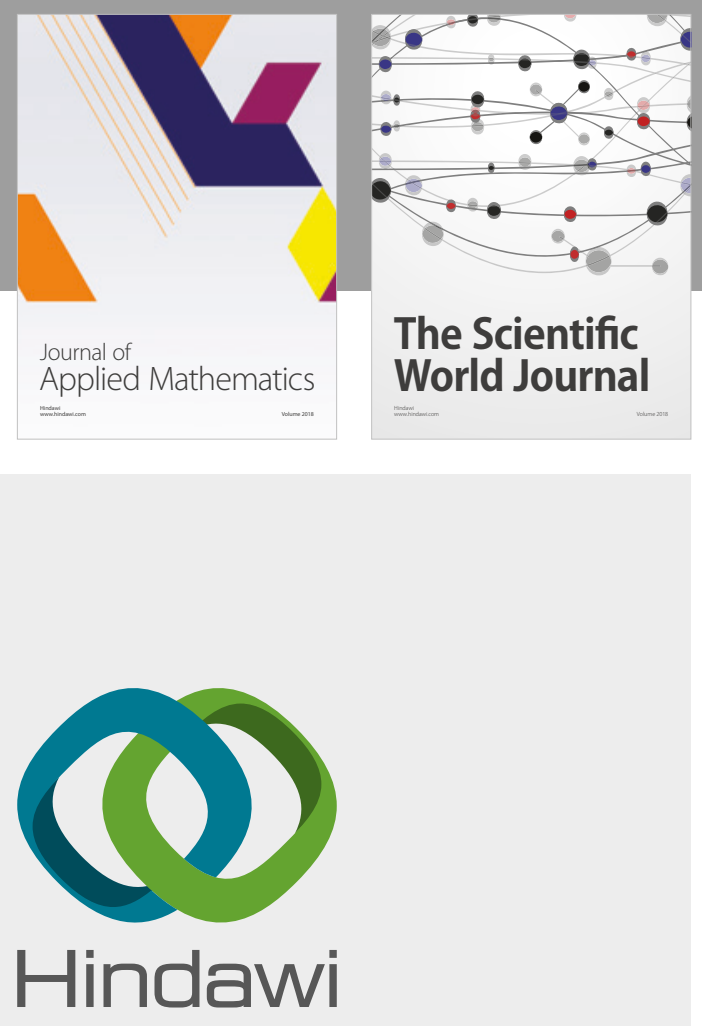

Submit your manuscripts at

www.hindawi.com

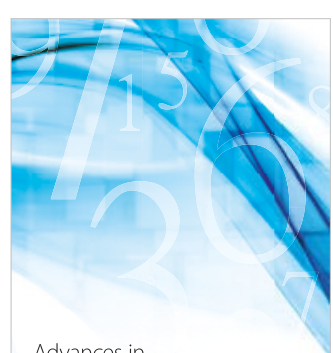

Advances in
Numerical Analysis
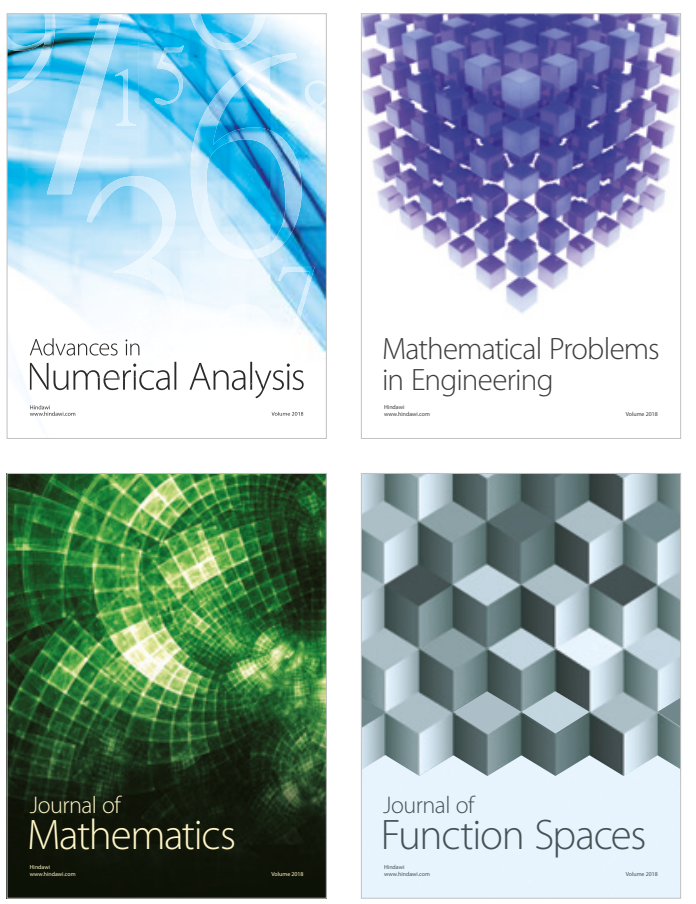

Mathematical Problems in Engineering

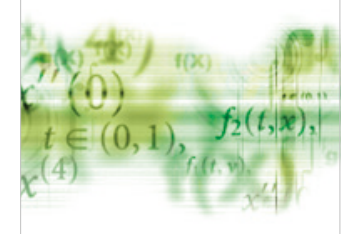

International Journal of

Differential Equations

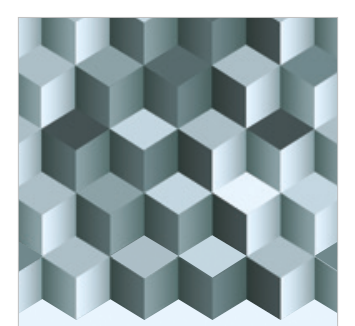

Journal of

Function Spaces

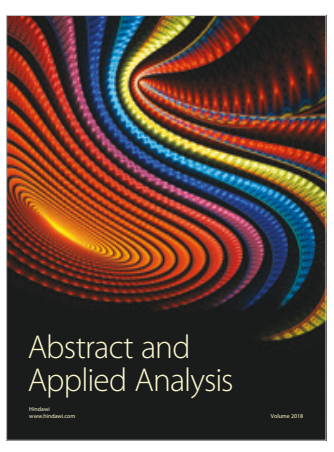

The Scientific

World Journal

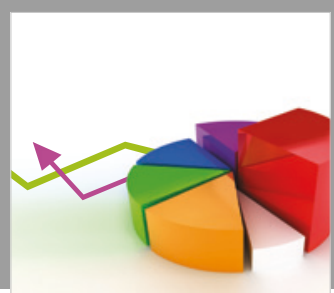

Journal of

Probability and Statistics
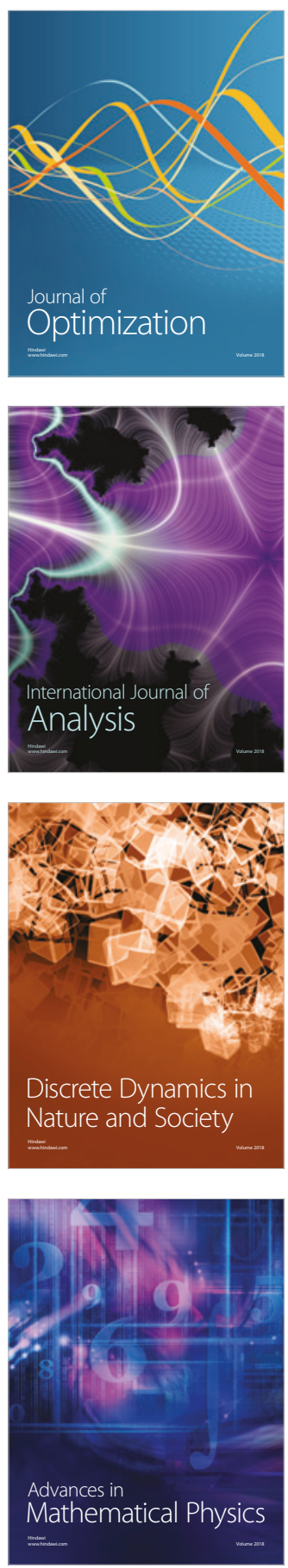